\title{
Error-Resilient Video Coding Performance Analysis of Motion JPEG 2000 and MPEG-4
}

\author{
Frederic Dufaux and Touradj Ebrahimi
}

\author{
Signal Processing Institute \\ Swiss Federal Institute of Technology (EPFL) \\ CH-1015 Lausanne, Switzerland \\ Frederic.Dufaux@epfl.ch \\ Touradj.Ebrahimi@epfl.ch
}

\author{
Emitall S.A. \\ Rue du Théâtre 5 \\ CH-1820 Montreux, Switzerland \\ Frederic.Dufaux@emitall.com \\ Touradj.Ebrahimi@emitall.com
}

\begin{abstract}
The new Motion JPEG 2000 standard is providing with some compelling features. It is based on an intra-frame wavelet coding, which makes it very well suited for wireless applications. Indeed, the state-of-the-art wavelet coding scheme achieves very high coding efficiency. In addition, Motion JPEG 2000 is very resilient to transmission errors as frames are coded independently (intra coding). Furthermore, it requires low complexity and introduces minimal coding delay. Finally, it supports very efficient scalability. In this paper, we analyze the performance of Motion JPEG 2000 in errorprone transmission. We compare it to the well-known MPEG-4 video coding scheme, in terms of coding efficiency, error resilience and complexity. We present experimental results which show that Motion JPEG 2000 outperforms MPEG-4 in the presence of transmission errors.
\end{abstract}

\section{INTRODUCTION}

The Joint Photographic Experts Group (JPEG) has recently produced a new standard for still image coding, referred to as JPEG 2000 [1]. JPEG 2000 not only provides with a state-of-the-art wavelet coding scheme outperforming previous techniques such as JPEG, but it also offers a number of features highly desired in multimedia applications. Among these features, we can mention progressive transmission by resolution or quality, lossy to lossless progressive compression, random code stream access and processing, continuous-tone and bi-level compression, and robustness to transmission errors.

Motion JPEG 2000 is an extension of JPEG 2000 for the coding of video sequences [2]. In this paper, we study its performance for the transmission of video over low bit rate error-prone wireless channels. In particular, we carry out a comparison with the well-known MPEG-4 standard.

Basically, Motion JPEG 2000 consists of the intra-frame coding of each frame using JPEG 2000. As a consequence, Motion JPEG 2000 does not exploit temporal redundancies. However, the wavelet coding technique significantly outperforms techniques using block-based Discrete Cosine Transform (DCT). Furthermore, because frames are coded independently, the damaging impact of transmission errors does not propagate across consecutive frames. In addition, intra-frame coding requires a low computational complexity and a low buffer memory size. Moreover, due to the precise post-compression rate control, minimal coding delay is introduced. Finally, Motion JPEG 2000 also provides with important features such as resolution and quality scalability.

As a reference for our performance analysis, we use the state-of-the-art MPEG-4 standard [3]. In contrast with Motion JPEG 2000, MPEG-4 is based on a motion compensated block-based DCT. Temporal redundancies are exploited by the use of motion compensation and inter-frame coding, hence achieving high coding efficiency. However, motion estimation and compensation require a high computational complexity. In addition, the temporal prediction loop 
introduces dependencies between coded frames. Therefore, the negative impact of transmission errors propagates across several frames. Finally, due to the intra-/inter-frame coding structure and the inherently inaccurate rate control, coding delay is introduced.

A number of publications have already addressed the performance of JPEG 2000 and MPEG-4. In [4], JPEG 2000 is reviewed and its performance analyzed and evaluated. A review of error resilience tools in JPEG 2000 and MPEG-4 is presented in [5], along with a performance comparison for still image coding. The error resilience tools in MPEG-4 have also been reviewed in [6]. In [7], the issue of evaluating video quality in mobile applications has been discussed, along with the presentation of subjective results for Motion JPEG 2000 and MPEG-4. A preliminary version of our study can also be found in [8].

In this paper, we propose a thorough comparison of the performance of Motion JPEG 2000 and MPEG-4. In particular, we investigate intra- and inter-frame coding in the context of error-prone transmission. Indeed, compression and error resilience define contradictory requirements. In other words, the higher the compression, the more important the resulting bits are, and hence a highly compressed bit stream is more sensitive to transmission errors. Taking into account the above observations, we carry out a performance evaluation of Motion JPEG 2000 and MPEG-4 in the framework of video transmission over low bit rate error-prone wireless channels. The performance is evaluated not only in terms of objective and subjective quality, but also computational complexity and coding delay.

This paper is structured as follow. In Sec. 2, we first review and assess the main characteristics of Motion JPEG 2000 and MPEG-4. In Sec. 3, we describe the error resilient coding tools in the two coding schemes. We discuss the simulation setup and the test conditions in Sec. 4. We present experimental results in Sec. 5. Finally, we draw conclusions in Sec. 6.

\section{CONSIDERATIONS ON MOTION JPEG 2000 AND MPEG-4}

While it is beyond the scope of this paper to review in details Motion JPEG 2000 and MPEG-4, in this section we give some general considerations and assessments of the two coding schemes. For a more detailed presentation of JPEG 2000 and MPEG-4, the reader is referred to [1] and [3] respectively.

Motion JPEG 2000 is based on an intra-frame wavelet coding technique, where as MPEG-4 is using block-based motion compensated DCT coding. Because it relies solely on intra-frame coding, Motion JPEG 2000 is sub-optimal in terms of coding efficiency. The wavelet coding results mainly in blur and ringing artifacts. Conversely, MPEG-4 is achieving high coding efficiency. However, the DCT coding is characterized by block and ringing artifacts.

In terms of error resiliency, Motion JPEG 2000 has the upper hand over MPEG-4, as the impact of transmission errors is limited to a single frame and does not propagate to subsequent frames.

As motion estimation and compensation are two computationally intensive tasks, MPEG-4 is more complex than Motion JPEG 2000. This is especially true at the encoding side.

Motion JPEG 2000 makes use of an interesting post-compression rate control technique. As a direct consequence, a precise target bit rate can be achieved, hence introducing minimal coding delays. Conversely, MPEG-4 uses the classical paradigm of rate control based on modeling and feedback, which is inherently inaccurate. Furthermore, the intra-/interframe coding structure (I-, P- and B-frames) gives different importance to frames. As a result, bit rate is often fluctuating greatly from one frame to another, resulting in increased buffering and coding delay.

Last but not least, the multi-resolution wavelet coding and embedded bit stream in Motion JPEG 2000 allows for a very efficient resolution or quality scalability. Again, Motion JPEG 2000 has clearly the upper hand over MPEG-4 in this respect.

The above considerations are summarized in Table 1. This paper aims at quantifying more precisely some of these general considerations. 


\begin{tabular}{|l|l|l|}
\hline & MJ2 & MP4 \\
\hline Intra-coding & wavelet & DCT \\
\hline Inter-coding & no & yes \\
\hline coding efficiency & low & high \\
\hline main artifacts & blur, ringing & block, ringing \\
\hline error resilience & high & low \\
\hline complexity & low & high \\
\hline rate control & accurate & inaccurate \\
\hline coding delay & low & high \\
\hline scalability & efficient & inefficient \\
\hline
\end{tabular}

Table 1: Main characteristics of Motion JPEG 2000 (MJ2) and MPEG-4 (MP4).

\section{ERROR RESILIENT CODING}

Video transmission over wireless networks is becoming more pervasive. Due to the potentially high impact of transmission errors on subjective quality, error resilient coding tools have been included in the Motion JPEG 2000 and MPEG-4 standards.

Compression aims at removing the redundancies in the bit stream in order to squeeze the amount of information to represent a video sequence. Conversely, error resilience consists in adequately adding redundancies in the bit stream in order to limit the impact of transmission errors. It is therefore obvious that these two procedures imply contradictory requirements. One aim of this paper is to investigate this trade-off.

Most coding schemes rely on the principle of Variable Length Codes (VLC). While they are efficient to code data, VLC are especially sensitive to transmission errors. More precisely, in the presence of errors, the decoder loses synchronization and becomes unable to decode further. As a result, not only the part of the bit stream where an error occurs is lost, but also all the data until the next position where the decoder is able to resynchronize, as illustrated in Figure 1.

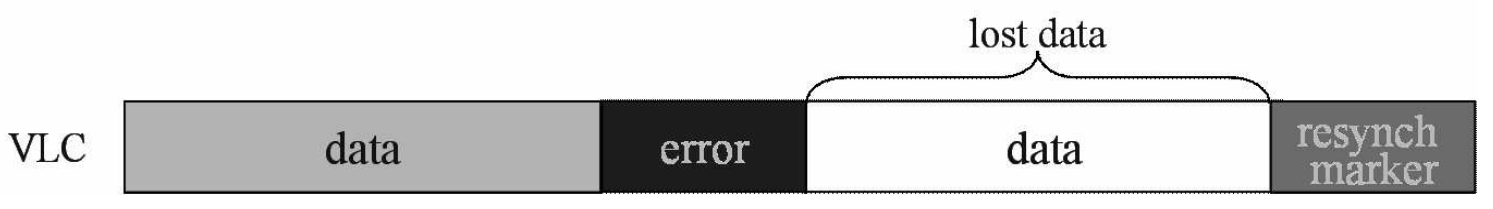

Figure 1: Impact of transmission errors on variable length codes.

In addition, if the decoding of a part of the bit stream depends on the correct decoding of previous parts of the bit stream, the effect of transmission errors will spread. More precisely, all parts of the bit stream which depend on the corrupted data will also be lost. Therefore, it is desirable to have coded units which can be independently decoded.

The purpose of the error resilient tools in Motion JPEG 2000 and MPEG-4 is to contain the impact of transmission errors. This is achieved by three means: detecting the occurrence of errors, concealing the erroneous data, and re-synchronizing the decoder. Hereafter, we review the error resilient tools in Motion JPEG 2000 and MPEG-4. A thorough description is also given in [5][6].

\subsection{ERROR RESILIENT TOOLS IN MOTION JPEG 2000}

The first and foremost advantage of Motion JPEG 2000 is that it is based on intra-frame coding. As all frames are coded independently from one another, transmission errors in one frame do not propagate to subsequent frames. This is the most important difference when compared to MPEG-4. 
Furthermore, Motion JPEG 2000 is relying on both resynchronization markers and data partitioning to limit the damage of transmission errors. More specifically, the code stream is composed of independently coded units or packets. This technique is commonly referred to as data partitioning. Each packet corresponds to a quality layer, a resolution, a component and a precinct. In addition, resynchronization markers can be optionally inserted in front of every packet, as illustrated in Figure 2. These markers are special codes which can be unequivocally recognized by the decoder, enabling the latter to resynchronize in the presence of errors.

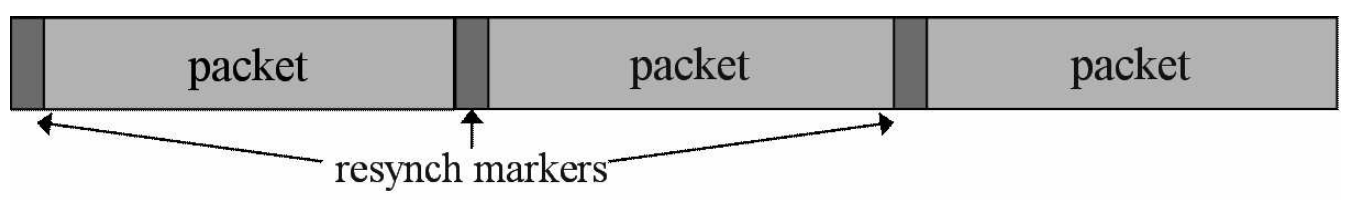

Figure 2: Resynchronization markers in Motion JPEG 2000.

The wavelet coefficients are partitioned into code-blocks, each code-block being independently coded. The coding is performed using an MQ arithmetic coder. A number of options can be used to strengthen its robustness to errors. The arithmetic coder can be terminated and the contexts can be reset after each coding pass. Additionally, a segment marker can be encoded at the end of each coding pass. In this case, if the segment marker is not correctly decoded at the decoder side, an error is flagged in the preceding coding pass.

However, whereas the above error resilient tools are only limiting the impact of transmission errors, they do not attempt to correct them. Furthermore, they do not deal with the occurrence of transmission errors in the image header, even though it is the most important part of the code stream. As a consequence, JPEG committee has also started a new work item, referred to as JPWL [9] to further improve the performance of JPEG 2000 over wireless networks. JPWL is notably addressing the protection of the image header, joint source-channel coding, unequal error protection, and data interleaving.

\subsection{ERROR RESILIENT TOOLS IN MPEG-4}

MPEG-4 is based on inter-frame coding. The correct decoding of a predictive frame ( $\mathrm{P}$ or B frames) depends on the truthful decoding of the reference frames (e.g. previous I or P frames). As a direct consequence, transmission errors can affect several frames. This is the most significant difference when compared to Motion JPEG 2000.

MPEG-4 is also using resynchronization markers. More specifically, the code stream is divided into video packets, in front of which resynchronization markers can be inserted, as depicted in Figure 3. The video packets consist of an integral number of macro-blocks. In a preferred operating mode, resynchronization markers are placed periodically.

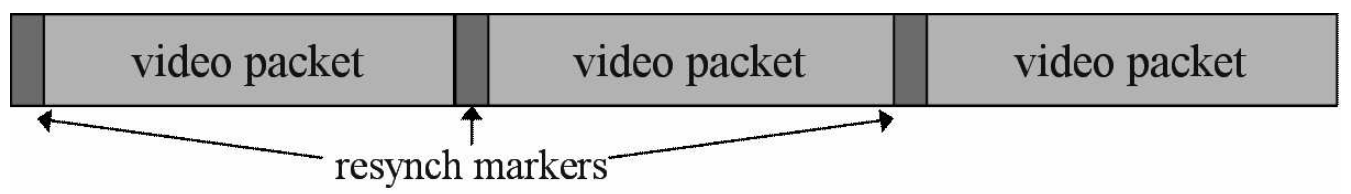

Figure 3: Resynchronization markers in MPEG-4.

MPEG-4 is supporting two distinct syntaxes. In the first case, the DCT coefficients, the motion vectors, and the information about macro-blocks coding mode are coded together in a combined syntax. This syntax is the most efficient in terms of coding, but is fragile in the presence of errors. Indeed, when an error occurs, all the data is likely to be lost.

The second syntax is based on the principle of data partitioning. More precisely, all the motion vectors are regrouped together, and similarly all the DCT coefficients are regrouped together. The bit stream is then formed as illustrated in Figure 4. The macro-block number (MB no) is followed by the quantization parameter (QP), all the motion data, a motion boundary marker (MBM) and finally all the DCT data. The MBM marker is used to validate the correct decoding 
of the motion data. If the DCT data is corrupted, all motion data can still be correctly decoded. Conversely, if the motion data contains errors, all the DCT data can still be recovered.

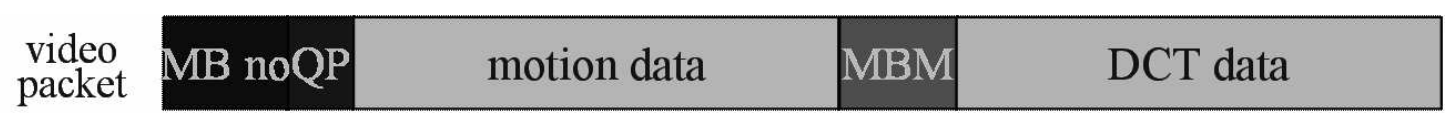

Figure 4: Data partitioning syntax.

We have already discussed the sensitivity of VLC to transmission errors. To circumvent this problem, MPEG-4 is giving the option to use Reversible Variable Length Codes (RVLC). Using RVLC, each codeword is identically decodable forward and backward. In the occurrence of an error, the decoder skips until the next resynchronization marker and then decodes the bit stream in the backward direction. As illustrated in Figure 5, more data can be recovered compared to the case of VLC.

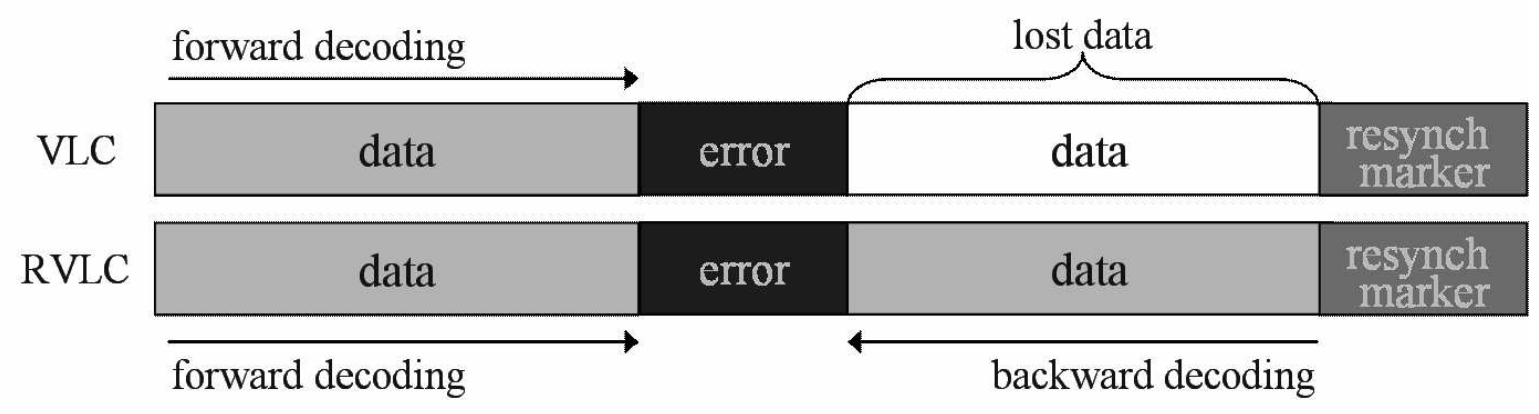

Figure 5: Reversible variable length codes.

Finally, as the picture header contains critical information such as the spatial resolution of a frame, the picture type and the time stamp, its loss results in the entire frame being dropped. To decrease the risk of losing the header, MPEG-4 has a mechanism to repeat the header information, referred to as Header Extension Code (HEC).

\section{SIMULATI ON ENVIRONMENT}

In this section, we discuss the simulation environment to compare the performance of Motion JPEG 2000 and MPEG-4. We first introduce the system used to simulate the transmission of video over a WCDMA wireless channel. We then describe the test sequences used in our experiments, along with the test conditions. And finally, we discuss the methodology to evaluate the results.

\subsection{SIMULATION SYSTEM}

In order to simulate the transmission of video sequences over a WCDMA wireless channel, we use the system depicted in Figure 6. The input video sequence is first encoded. In our case, we use either Motion JPEG 2000 or MPEG-4. More specifically, we use the Kakadu software [10] for Motion JPEG 2000 and the MoMuSys reference software [11] for MPEG-4. Source encoding is then followed by multiplexing and packetization of the bit stream using H.223. Transmission errors are randomly injected using bit error patterns representative of WCMDA [12]. To retrieve the output video sequence, the dual operations are performed, namely H.223 demultiplexing and source decoding. 


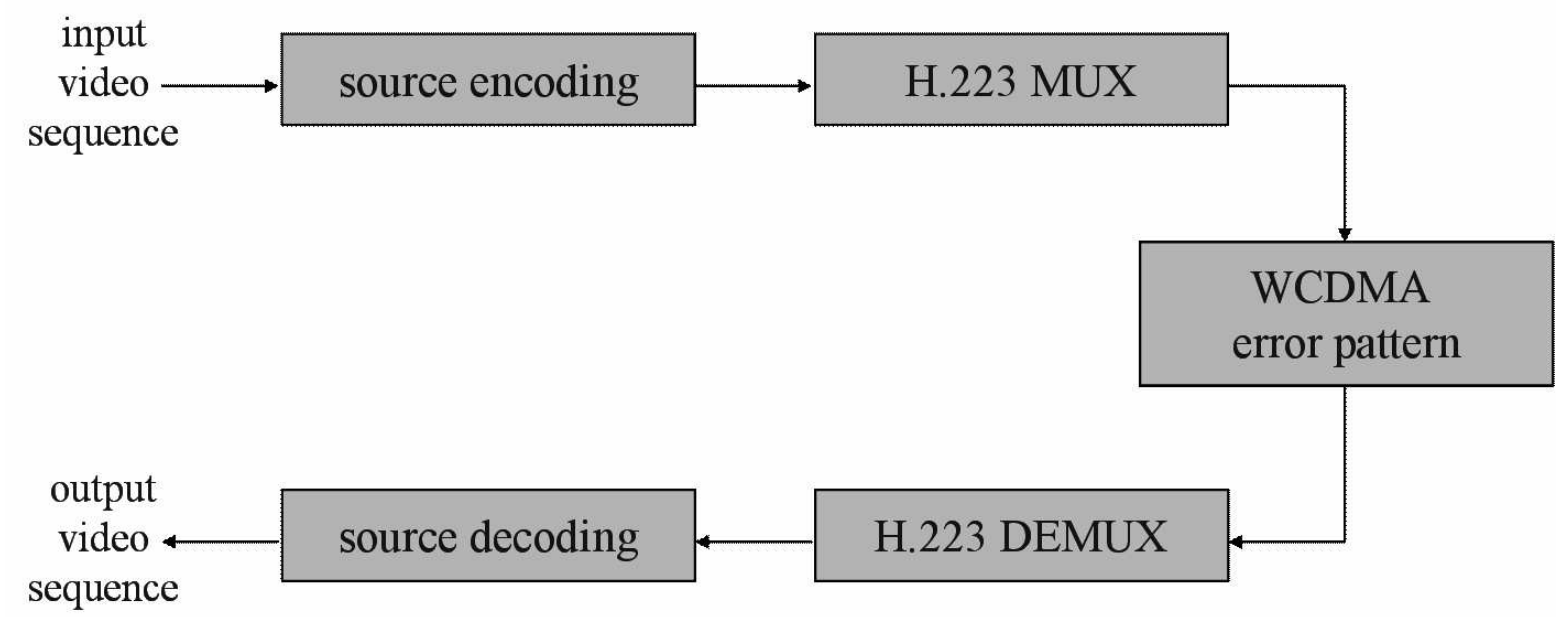

Figure 6: Simulation system for video transmission over a WCDMA wireless channel.

Due to the random nature of transmission errors, a large number of trials are run. Each trial consists in applying a different random circular shift to the same bit error pattern. Final figures are then obtained by averaging results over all the trials.

\subsection{TEST SEQUENCES}

We selected 9 video sequences as illustrated in Figure 7. The sequences have been chosen to cover a wide range of content, and are characterized by motion ranging from slow to fast. Consequently, the sequences span a wide range of coding complexity.
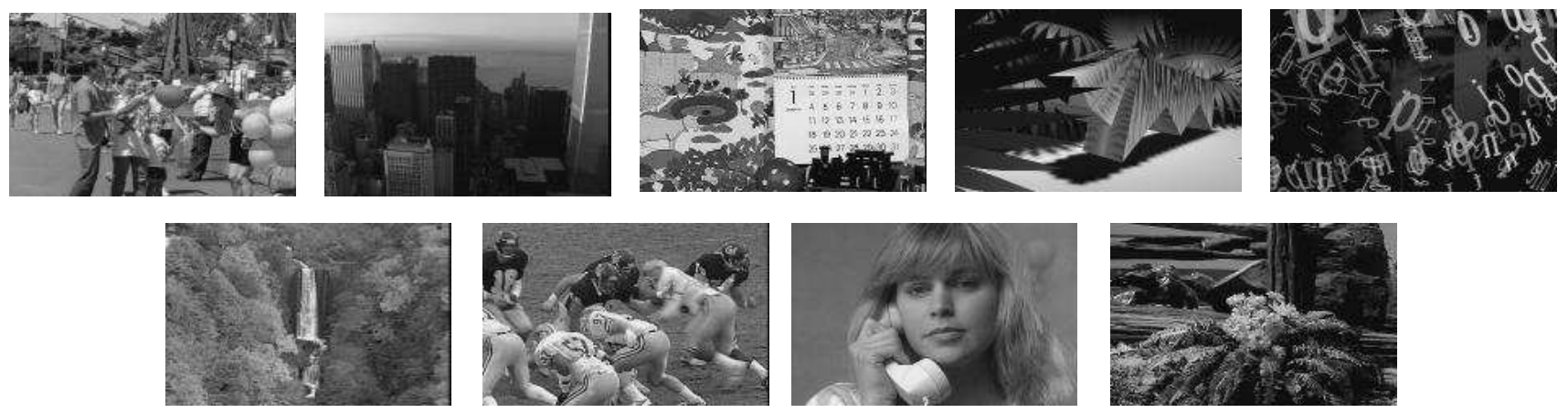

Figure 7: Test sequences: Balloons, New York, Mobile, Animals, Letters, Waterfall, Football, Suzie, and Tempest.

\subsection{TEST CONDITIONS}

Taking into account the constraints of wireless applications, the sequences are encoded at $128 \mathrm{~kb} / \mathrm{s}$ henceforth implying low spatial and temporal resolutions. More specifically, the sequences are encoded in QCIF format with a frame rate of 6 fps.

We run simulations in the two cases when transmission errors are absent or present. In the latter case, we use a Bit Error Rate (BER) of 1e-4, and the results are obtained by averaging over 100 trials for each sequence. 
For Motion JPEG 2000, the Kakadu software [10] is used. All the error resilience tools, as described in Sec. 3.1, are applied. The rate control is applied on a per frame basis. In other words, each frame is allocated the same bit budget.

As far as MPEG-4 is concerned we are using the MoMuSys reference software [11] with options corresponding to the Simple Profile of Part 2. Indeed, we believe this profile is the most appropriate for wireless applications in terms of coding efficiency and complexity, resulting therefore in a meaningful comparison. All the error resilience tools, as described in Sec. 3.2, are applied. The VM4 rate control is used in order to achieve the target bit rate. We evaluate three different intra-/inter-frame coding structures:

- IPPPPP: The first frame is intra-coded; all subsequent frames are inter-frame coded (P-frames). This structure offers the highest coding efficiency, but is more sensitive to transmission errors. Furthermore, it does not allow for random access into the bit stream.

- IPPIPP: Intra refresh is applied periodically; all other frames are inter-frame coded (P-frames). In our case, the intra refresh is executed every second. This structure tends to a slightly lower coding efficiency, but is more resistant to transmission errors. Furthermore, it allows for random access into the bit stream.

- IIIIII: All frames are intra-coded. As a result, coding efficiency is lower than in the two previous cases. However, error resilient is much higher.

It is obvious that the performance of Motion JPEG 2000 and MPEG -4 depends on the specific encoder design. However, we consider that the two above reference implementations lead to representative results. Therefore, it gives a good indication of the performance of the respective coding schemes.

\subsection{METHODOLOGY TO EVALUATE THE RESULTS}

We consider separately the performance in the two cases without and with transmission errors. Objective quality is measured by Peak-Signal-to-Noise-Ratio (PSNR). However, it is well-known that PSNR is not always a good measure of perceptual quality. This is especially true when the artifacts are very different. Therefore, we also used subjective metrics obtained with Genista's Video PQoS software [13]. More specifically, we report results for perceptual metrics evaluating three types of artifacts, namely block, blur and noise. Finally, we inspect visually the decoded sequences.

Computational complexity is another important property. For this purpose, we evaluate complexity by profiling the software at run time. Nevertheless, these figures depend on the optimization level of the respective codes.

The last important property is the amount of deviation in the bit rate, hence requiring a buffer which introduces coding delay. To illustrate this property, we show the variation in the number of bits consumed by each coded frame.

\section{SIMULATION RESULTS}

In this section, we report the results of our experiments comparing Motion JPEG 2000 and MPEG-4, in terms of objective and subjective quality, complexity and coding delay.

\subsection{OBJECTIVE RESULTS - PSNR}

We first consider objective results. The performance depends strongly on the content and more specifically on the amount of motion in the sequence.

Figure 8 shows PSNR results for the sequence Suzie for the two cases without and with transmission errors. We can see that in the absence of transmission errors, typically MPEG-4 with IPPPPPP and IPPIPP structures is slightly outperforming Motion JPEG 2000. The performance of MPEG-4 with IIIIII structure is significantly lower. We can also observe that due to the rate control, the PSNR of MPEG-4 with inter-frame coding (IPPPPPP and IPPIPP) is fluctuating widely, whereas it is nearly constant for Motion JPEG 2000 and MPEG-4 with intra-frame coding (IIIIII). 
In the presence of transmission errors, Motion JPEG 2000 is gaining the upper hand over MPEG-4. The drop due to transmission errors is the most significant for MPEG-4 with IPPPPPP structure. In this case, the IPPIPP structure is the best one for MPEG-4.
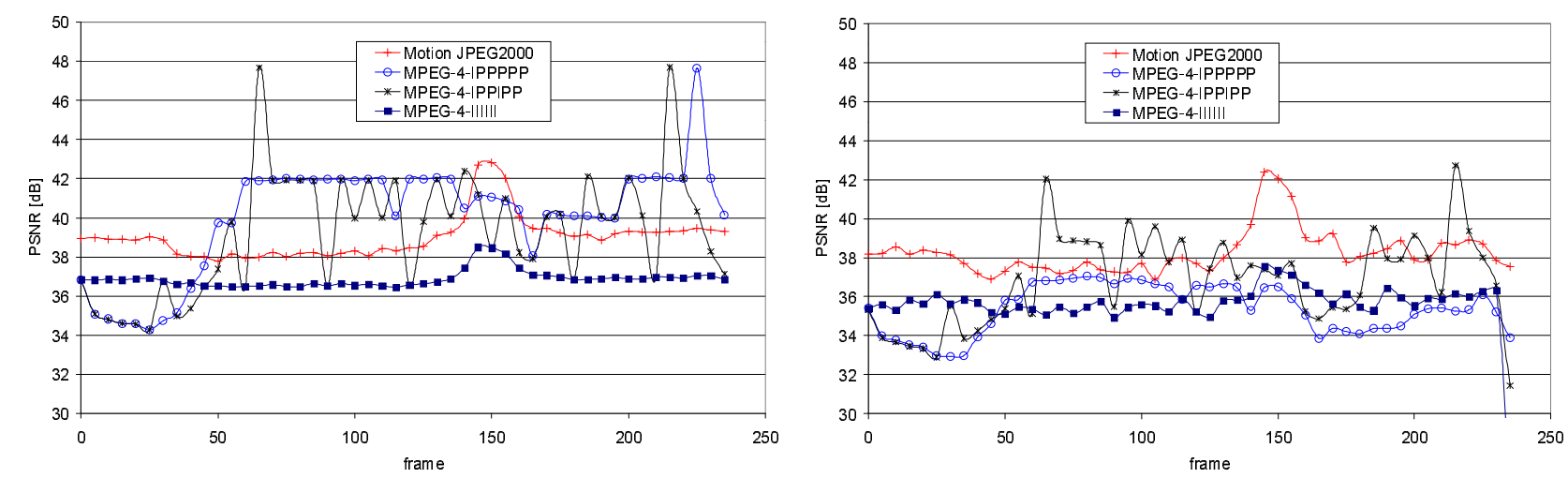

Figure 8: PSNR results for Suzie, left: without transmission errors, right: with transmission errors (BER=1e-4).

Results for all test sequences are summarized in Table 2 and Table 3 for the two cases without and with transmission errors. It can be observed that performance is strongly content-dependent. Without transmission errors, MPEG-4 (IPPPPP and IPPIPP) is typically superior on sequences with low to moderate motion, whereas Motion JPEG 2000 is best on sequences with fast motion.

In the presence of errors, Motion JPEG 2000 is outperforming all variants of MPEG-4. MPEG-4 with IPPIPP or IPPPPP is experiencing a drop due to errors of 1.5 and $3 \mathrm{~dB}$ respectively. Limiting MPEG-4 to intra-coding is not a solution. Indeed, while MPEG-4 with IIIIII structure is resisting well to errors, its performance is consistently the worse.

\begin{tabular}{|c|c|c|c|c|}
\hline sequence & MJ2 & MP4-IPPPPP & MP4-IPPIPP & MP4-IIIIII \\
\hline Balloons & 27.77 & 27.55 & 27.49 & 27.05 \\
\hline New York & 35.63 & 37.40 & 36.58 & 34.76 \\
\hline Mobile & 23.11 & 26.43 & 25.47 & 22.62 \\
\hline Animals & 34.28 & 37.71 & 36.28 & 32.88 \\
\hline Letters & 27.32 & 26.16 & 26.17 & 26.48 \\
\hline Waterfall & 29.92 & 32.87 & 32.06 & 29.16 \\
\hline Football & 32.98 & 31.37 & 31.61 & 32.02 \\
\hline Suzie & 39.00 & 40.11 & 39.30 & 36.87 \\
\hline Tempest & 27.07 & 29.47 & 28.72 & 26.15 \\
\hline average & $\mathbf{3 0 . 7 9}$ & $\mathbf{3 2 . 1 2}$ & $\mathbf{3 1 . 5 2}$ & $\mathbf{2 9 . 7 8}$ \\
\hline MJ2K gain & & $\mathbf{- 1 . 3 3}$ & $\mathbf{- 0 . 7 3}$ & $\mathbf{1 . 0 1}$ \\
\hline
\end{tabular}

Table 2: PSNR results without transmission errors.

\begin{tabular}{|c|c|c|c|c|}
\hline sequence & MJ2 & MP4-IPPPPP & MP4-IPPIPP & MP4-IIIIII \\
\hline Balloons & 27.41 & 25.76 & 26.44 & 26.47 \\
\hline New York & 35.06 & 32.48 & 34.25 & 33.49 \\
\hline Mobile & 22.87 & 24.68 & 24.67 & 22.29 \\
\hline Animals & 33.63 & 32.28 & 33.36 & 31.71 \\
\hline Letters & 26.90 & 24.81 & 25.34 & 25.95 \\
\hline
\end{tabular}




\begin{tabular}{|c|c|c|c|c|}
\hline Waterfall & 29.62 & 31.06 & 31.29 & 28.76 \\
\hline Football & 32.48 & 29.39 & 30.21 & 31.28 \\
\hline Suzie & 38.25 & 35.35 & 36.82 & 35.58 \\
\hline Tempest & 26.69 & 27.02 & 27.75 & 25.73 \\
\hline average & $\mathbf{3 0 . 3 2}$ & $\mathbf{2 9 . 2 0}$ & $\mathbf{3 0 . 0 1}$ & $\mathbf{2 9 . 0 3}$ \\
\hline MJ2K gain & & $\mathbf{1 . 1 2}$ & $\mathbf{0 . 3 1}$ & $\mathbf{1 . 2 9}$ \\
\hline
\end{tabular}

Table 3: PSNR results with transmission errors (BER=1e-4).

\subsection{SUBJECTIVE RESULTS - PERCEPTUAL METRICS AND VISUAL QUALITY}

Motion JPEG 2000 and MPEG-4 generate very different artifacts. The reason is the fundamentally different underlying technology, intra-frame wavelet in the first case, and motion compensated DCT in the second case. More specifically, wavelet typically introduces blur and ringing artifacts, where as DCT is characterized by block and ringing artifacts.

In Figure 9, we show results obtained using three perceptual metrics developed by Genista [13] to measure block, blur and noise artifacts respectively. The results correspond to the sequence Balloons with transmission errors. The vertical axis is in arbitrary units: a small value signifies the artifact is not detected, while a large value indicates the artifact is very strong. It can be observed that all variants of MPEG-4 exhibit strong block artifacts, as expected. Obviously, this artifact is not detected for Motion JPEG 2000. In terms of blur, all coding schemes have approximately the same performance. Finally, for the amount of noise, Motion JPEG 2000 is again outperforming all variants of MPEG-4.
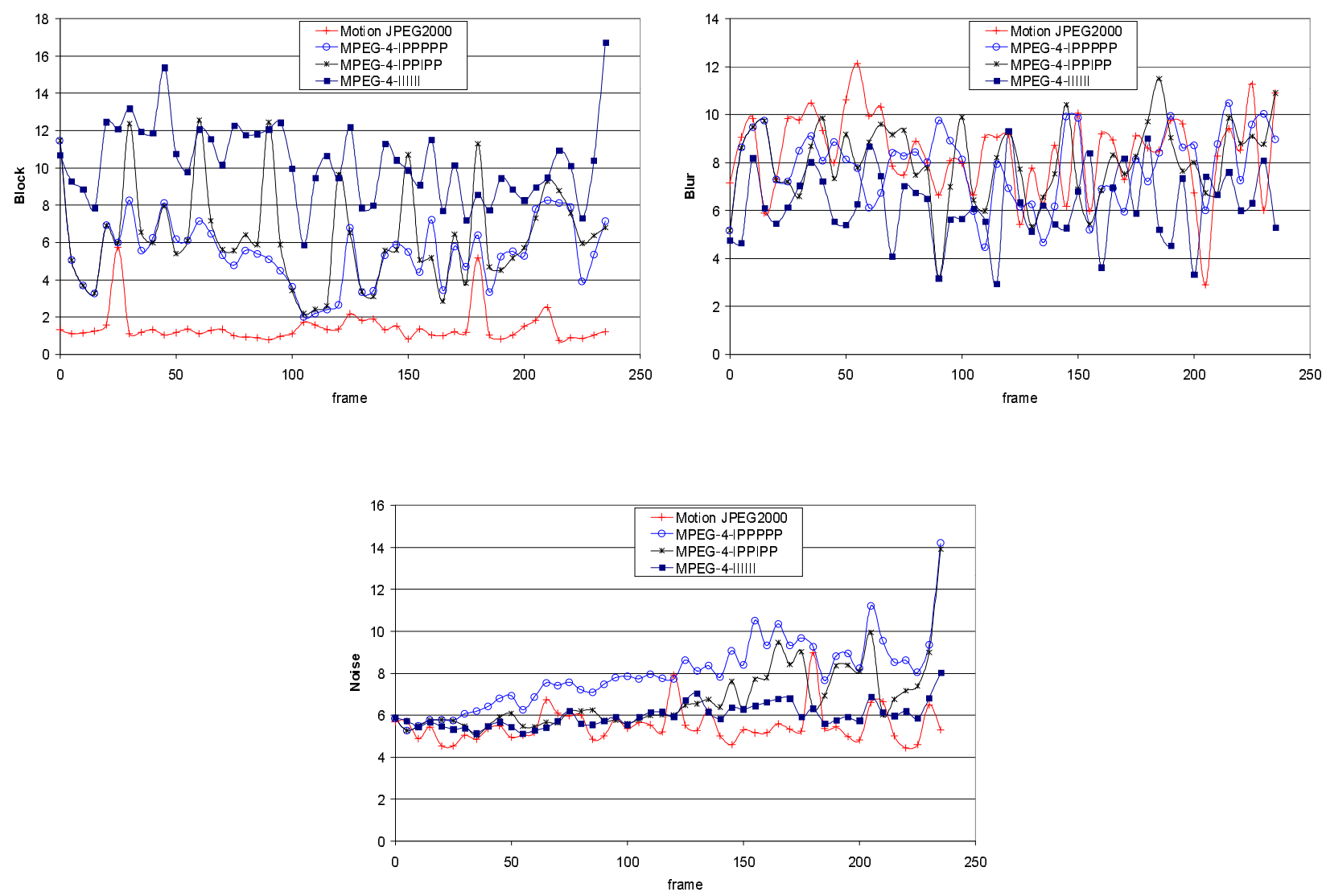

Figure 9: Perceptual results for Balloons, top-left: block, top-right: blur, bottom: noise. 
To complement the above subjective results, sample decoded frames from the sequences Balloons and Suzie are shown in Figure 10, for the case with transmission errors (BER=1e-4). While artifacts are due to both the compression and the transmission errors, we show examples where artifacts due to transmission errors dominate. As can be observed, transmission errors tend to introduce hazy waves in Motion JPEG 2000. Conversely, they generate strong block artifacts (e.g. blocks of strange color or texture) in MPEG-4, which are visually more annoying.
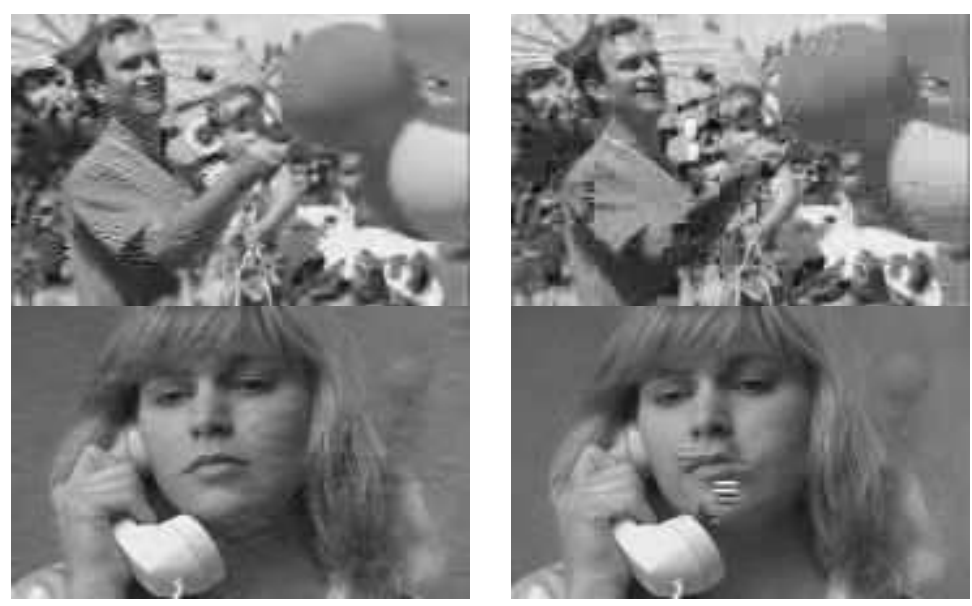

Figure 10: Sample frames (top: Balloons, bottom: Suzie, left: Motion JPEG 2000, right. MPEG-4).

Furthermore, the temporal evolution of these artifacts is very different. More specifically, in Motion JPEG 2000 only the specific frame which has been corrupted exhibits distortions. In other words, the artifacts are very transient. On the other hand, the distortions persist over several frames in MPEG-4, until an intra refresh takes place. Finally, due to the influence of intra-refresh and rate control, quality varies more widely along time in MPEG-4. For these reasons, the artifacts are significantly more visible and disturbing in MPEG-4 when compared to Motion JPEG 2000.

\subsection{COMPUTATIONAL COMPLEXITY}

We compare the computational complexity of Motion JPEG 2000 and MPEG-4, for both encoding and decoding. The results have been obtained by profiling the software at run time, and are shown in Table 4 . These results depend therefore on the optimization level of the respective codecs. The figures for MPEG-4 have been obtained with the Microsoft reference software, as it is much faster than the MoMuSys implementation.

\begin{tabular}{|l|l|l|}
\hline CPU [ms/frame] & MJ2 & MP4 \\
\hline Encoder & 88 & 390 \\
\hline Decoder & 38 & 45 \\
\hline
\end{tabular}

Table 4: CPU for encoding and decoding.

As can be observed, MPEG-4 is more complex than Motion JPEG 2000. The different is due to the complexity of the motion estimation and compensation used in MPEG-4. Quantitatively, the complexity for MPEG-4 encoding is approximately 5 times larger as for Motion JPEG 2000. The difference is small for decoding.

\subsection{BIT RATE VARIATION}

Figure 11 shows the variation in the number of bits consumed for each coded frame. It can be seen that due to its very accurate post-compression rate control, Motion JPEG 2000 results in an absolutely constant bit rate. On the other hand, 
the inaccurate rate control of MPEG-4 based on model and feedback, combined with the inter-/intra-frame coding structure results in wide variations in the number of coded bits per frame. Consequently, MPEG-4 requires buffering which introduces coding delay.

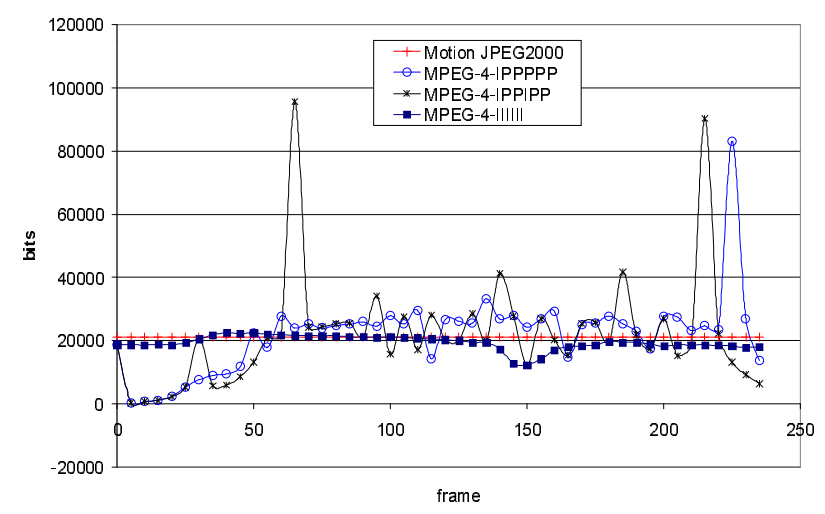

Figure 11: Bit consumption variation for Suzie.

\section{CONCLUSIONS}

In this paper, we have investigated the performance of Motion JPEG 2000 and compared it to MPEG-4. We have shown the strong performance of Motion JPEG 2000, especially in the case of error-prone video transmission over wireless channels. Motion JPEG 2000 has the upper hand over MPEG-4 when errors occur, with gains ranging from 0.31 to 1.29 dB. In addition, Motion JPEG 2000 offers other compelling features such as lower complexity, better scalability and low coding delay. In conclusion, Motion JPEG 2000 is very well suited for wireless applications.

\section{ACKNOWLEDGMENT}

EPFL's contribution to this work was partially supported by the European Project 2KAN. Please refer to http://www.2kan.org for more details about this project and its objectives. Authors acknowledge Kambiz Homayounfar from Genista Corporation for valuable inputs and insights to the work presented here.

\section{REFERENCES}

[1] D. Taubman and M. Marcellin, "JPEG 2000: Image Compression Fundamentals, Standards and Practice", Kluwer Academic Publishers, 2002.

[2] ISO/IEC 15444-3:2002, "Information technology - JPEG 2000 image coding system - Part 3: Motion JPEG 2000", 2002.

[3] T. Ebrahimi and F. Pereira, “The MPEG-4 Book”, Prentice Hall, 2002.

[4] D. Santa-Cruz, R. Grosbois and T. Ebrahimi, "JPEG 2000 performance evaluation and assessment", Signal Processing: Image Communication, vol. 17, no. 1, pp. 113-130, January 2002.

[5] I. Moccagatta, S. Soudagar, J. Liang and H. Chen, "Error-Resilient Coding in JPEG-2000 and MPEG-4", IEEE Journal on Selected Areas in Communications, vol. 18, no. 6, pp. 899-914, June 2000. 
[6] R. Talluri, "Error-resilient video coding in ISO MPEG-4 standard”, IEEE Commum. Mag., vol. 36, no. 6, pp. 112119, July 1998.

[7] S. Winkler and F. Dufaux, "Video Quality Evaluation for Mobile Applications", in SPIE Proc. Visual Communication and Image Processing, vol. 5150, Lugano, Switzerland, July 2003.

[8] F. Dufaux and T. Ebrahimi, "Motion JPEG 2000 for Wireless Applications", in First International Workshop on JPEG 2000, Lugano, Switzerland, July 2003.

[9] ISO/IEC JTC1/SC29/WG1 WG1N2696, “JPWL Scope and Requirements”, March 2002.

[10] http://www.kakadusoftware.com (v3.4).

[11] ISO/IEC JTC1/SC29/WG11 WG11N5550, "ISO/IEC 14496-7/DAM1 Optimized reference software for coding of audio-visual objects", March 2003.

http://mpeg.telecomitalialab.com/working_documents/mpeg-04/optimised_software/14496-7dam1.zip.

[12] ITU-T Study Group 16, “WCDMA Error Patterns at 128 and 384 kbps”, Technical Report Q15-G28, 1999.

[13] http://www.genista.com. 